\title{
Higher Spin Cosmology
}

\author{
Chethan KRISHNAN叫, Avinash RAJU呾, Shubho ROY吗 \\ and Somyadip THAKUR里 \\ ${ }^{a}$ Center for High Energy Physics \\ Indian Institute of Science, Bangalore, India
}

\begin{abstract}
We construct cosmological solutions of higher spin gravity in $2+1$ dimensional de Sitter space. We show that a consistent thermodynamics can be obtained for their horizons by demanding appropriate holonomy conditions. This is equivalent to demanding the integrability of the Euclidean boundary CFT partition function, and reduces to Gibbons-Hawking thermodynamics in the spin-2 case. By using a prescription of Maldacena, we relate the thermodynamics of these solutions to those of higher spin black holes in $\mathrm{AdS}_{3}$.
\end{abstract}

\footnotetext{
${ }^{1}$ chethandcts.iisc.ernet. in

${ }^{2}$ avinash@cts. iisc. ernet. in

${ }^{3}$ sroy@het . brown . edu

${ }^{4}$ somyadip@cts.iisc . ernet. in
} 


\section{Introduction}

In $2+1$ dimensions, pure gravity has no (perturbative) dynamics because curvature is completely rigid. But despite the lack of any gravitational attraction, gravity in $2+1$ dimensions is non-trivial - black holes solutions were discovered by Banados, Teitelboim and Zanelli (BTZ) [1] as quotients of $\mathrm{AdS}_{3}$ [2]. This fact makes $2+1 \mathrm{D}$ gravity an excellent theoretical laboratory for testing a variety nonperturbative issues in quantum gravity, without the added complications of curvature dynamics which play a huge role in higher dimensions. However, effort in this direction did not begin in earnest until the work of Witten [3] (see also [4]). He demonstrated that $2+1$-d gravity can be recast as a Chern-Simons gauge theory, with the gauge gauge group $S L(2, R) \times S L(2, R)$ when the cosmological constant $\Lambda$ is $<0$, and the gauge group $S L(2, C)$ when $\Lambda$ is $>0$.

The negative cosmological constant case drew a lot of attention, partly because that was the context in which the above mentioned BTZ black holes were discovered, but also because of the earlier work of Brown and Henneaux [5] who showed that the asymptotic symmetry algebra of $\mathrm{AdS}_{3}$ gravity is a Virasoro algebra. In fact, this latter result is now widely recognized as a precursor to the celebrated AdS-CFT duality [6] where a fully quantum theory of gravity in $\mathrm{AdS}_{d+1}$ is conjectured to have an equivalent description in terms of a conformal field theory supported on the boundary of $\mathrm{AdS}_{d+1}$. However, there was no such happy ending in the case of a positive cosmological constant, namely $\mathrm{dS}_{3}$. Although the counterpart to the BTZ black hole quotients were constructed in [7] (also see [8]), the fact that de Sitter is a cosmological spacetime with a spacelike boundary [9] has made the development of a consistent $\mathrm{dS} / \mathrm{CFT}$ proposal much more confusing. Various interesting attempts were made in [10, 11, 12, but there seems to be a fundamental difficulty in realizing de Sitter space in any kind of unitary quantum set up as a stable vacuum [13, 14, 15, 16, 17, 18.

On an entirely different theme, theories of interacting gauge fields with an infinite tower of higher spins $(s \geq 2)$ have been studied as a toy version of a full string theory $5^{5}$ by Fradkin and Vasiliev [20, 21, 22, 23, 24, 25], building on the early work of Fronsdal [26]. Higher spin theories in three dimensions, as demonstrated in [27] are considerably simpler than theories in higher dimensions due to absence of any local propagating degrees of freedom. In addition, it is possible to truncate the infinite tower of higher spin fields to spin, $s \leq N$. The complicated nonlinear interactions of the higher spin fields can be reformulated in terms of an $S L(N, R) \times S L(N, R)$ Chern-Simons gauge theory (for $\mathrm{AdS}_{3}$ case) or an $S L(N, C)$ Chern-Simons (for $\mathrm{dS}_{3}$ ). Therefore $2+1$ dimensional higher spin theories are a generalization of Chern- Simons gravity - one gets back to the spin-2 pure gravity theory when one sets $N=2$.

The aim of this paper is to construct cosmological solutions in higher spin $\mathrm{dS}_{3}$ gravity. We work specifically with the case where the rank of the gauge group, $N=3$. The solutions we construct are the higher spin generalizations of $\mathrm{dS}_{3}$ quotients such as Kerr- $\mathrm{dS}_{3}$ and quotient cosmology [7, 8, 28, and should be thought of as the de Sitter counterparts of the spin-3 charged $\mathrm{AdS}_{3}$ black hole solutions of [29, 30]. It has been shown recently by two of us that big-bang type singularities contained in quotient cosmologies in the purely $S L(2)$ sector of this higher spin theory can be removed by performing a spin-3 gauge transformation [28]. But the problem of constructing spin-3 charged cosmologies was left open. In this paper, we fill this gap and discuss the thermodynamics

\footnotetext{
${ }^{5}$ Since the latter has infinite dimensional gauge invariance, see the work by Sundborg [19].
} 
of their cosmological horizons.

The plan of the paper is the following. In Sec. 2, we recap the formulation of spin-3 field coupled to gravity in 2+1-dimensions as a Chern-Simons gauge theory with a non-compact gauge group, $S L(3, C)$. We fix all notations and conventions for the map between the second order variables (metric and spin-3 field) and the gauge connection here. We also review and discuss the variational principle for asymptotically de Sitter like connections in the gauge theory formulation. In Sec. 3, we first review pure gravity i.e. $S L(2, C)$ sector solutions, namely the Kerr de Sitter universe and the quotient cosmology. We do this both in the metric and gauge theory set-up. Then we construct higher spin extensions of these geometries by modifying their gauge connection and adding spin-3 charges in a manner consistent with the triviality of gauge connection holonomies along contractible cycles. These solutions are shown to contain cosmological horizons and, in the case of quotient cosmology, higher spin big bang/ big crunch like causal singularities. In the final section, Sec. 4, these holonomy conditions are shown to be necessary for the consistency of thermodynamics associated with cosmological horizons. These consistency conditions turn out to be identical to demanding integrability of a "boundary CFT partition function". Using a prescription of Maldacena [12, we relate thermodynamics of our solutions to those of higher spin $\mathrm{AdS}_{3}$ black holes. Our formulation gives the same results as the Gibbons-Hawking results when we restrict to spin-2 and work in the metric language.

\section{$2 S L(3, C)$ Chern-Simons formulation of Higher Spin $\mathbf{d S}_{3^{-}}$ gravity}

Here we quickly review the basics of the $S L(3, C)$ CS gauge theory generalizing Witten's construction [3] as presented in detail in [31, 28] (see also [32] for discussion on higher spins in $\mathrm{dS}_{3}$ ). One simply defines the higher spin (up to spin 3) theory i.e. an interacting theory of gravity and a spin 3 -field, by the action [27]

$$
I_{C S}[A]=\frac{k}{4 \pi d_{R}} \int_{M} \operatorname{Tr}\left(A d A+\frac{2}{3} A^{3}\right)-\frac{k}{4 \pi d_{R}} \int_{M} \operatorname{Tr}\left(\bar{A} d \bar{A}+\frac{2}{3} \bar{A}^{3}\right) .
$$

The constant $d_{R}=-2 \operatorname{Tr}\left(T_{0} T_{0}\right)$ is a characteristic of the representation size. Here the gauge field, $A$ is a complex $S L(3)$ matrix-valued one form. In a basis of $S L(3)$ matrices, $\left\{T_{a}, T_{a b} ; a, b=0,1,2\right\}$ as listed in [33], we can expand the gauge field as

$$
\begin{aligned}
A & =\left(\omega_{\mu}^{a}+\frac{i}{l} e_{\mu}^{a}\right) T_{a} d x^{\mu}+\left(\omega_{\mu}^{a b}+\frac{i}{l} e_{\mu}^{a b}\right) T_{a b} d x^{\mu} \\
& =\left(\omega_{\mu}+\frac{i}{l} e_{\mu}\right) d x^{\mu}, \omega_{\mu}=\omega_{\mu}^{a} T_{a}+\omega_{\mu}^{a b} T_{a b}, e_{\mu}=\omega_{\mu}^{a} T_{a}+\omega_{\mu}^{a b} T_{a b}
\end{aligned}
$$

Then, the more familiar metric and spin-3 field can be extracted from the (imaginary parts of the basis coefficients) of the gauge field [33]:

$$
g_{\mu \nu}=\frac{1}{2 !} \operatorname{Tr}\left(e_{\mu} e_{\nu}\right), \phi_{\mu \nu \lambda}=\frac{1}{3 !} \operatorname{Tr}\left(e_{(\mu} e_{\nu} e_{\lambda)}\right)
$$


while the three-dimensional Newton's constant (in units of the dS radius, $l$ ) is given by the ChernSimons level number,

$$
\frac{G_{3}}{l}=\frac{1}{4 i k}
$$

We work in the prevalent general relativity convention where, $8 G_{3}=1$. Since the gauge group $S L(3, C)$ is non-compact the Chern-Simons level number is not quantized.

Now lets consider the variation of the action (1). Generically a variation has a bulk (volume) piece, proportional to the equation of motion and boundary pieces supported on temporal and spatial boundaries,

$$
\delta I=\int d^{3} x(E . O . M)+\left.\int d^{2} x \pi_{\mu} \delta A^{\mu}\right|_{t_{i}} ^{t_{f}}+\left.\int d t d x^{j} \pi_{\mu}^{j} \delta A^{\mu}\right|_{x_{i, \text { min }}} ^{x_{i, \max }}
$$

To have a good variational principle one has to ensure that these boundary pieces vanish (onshell) by prescribing initial and final conditions and spatial boundary conditions. If the prescribed conditions do not lead to vanishing contribution for the boundary pieces of the variation, then one has to add supplementary boundary terms to the action to cancel these. One crucial point to be noted here in contrast with the AdS case is that the action (11) already defines a good variational principle without any supplementary boundary terms. This is because asymptotically deSitter spaces have closed spatial sections and the only boundary contributions are from future infinity $\left(t_{f} \rightarrow \infty\right)$ and at some time coordinate in the past $\left(t_{i}=\right.$ const). As the variational principle is usually defined with vanishing variations at the initial and final times,

$$
\left.\delta A\right|_{t_{i}, t_{f}}=0
$$

these boundary pieces vanish. However we shall not demand the future data to be fixed (i.e. $\left.\delta A\right|_{t_{f} \rightarrow \infty} \neq 0$ ) and look to set up a variational principle by demanding instead the conjugate momentum vanishes

$$
\left.\pi_{\mu}\right|_{t_{f} \rightarrow \infty} \rightarrow 0
$$

Such a variational principle will be made to appear natural in Sec. 4 where the close parallel between de Sitter and Anti de Sitter cases is brought out. This will often restrict us to a subclass of solutions which are specified by their future fall-off behaviors (which close under gauge transformations),

$$
\lim _{t \rightarrow \infty} A_{\mu} \sim t^{\alpha_{\mu}}
$$

for some real bounded exponent $\alpha_{\mu}$. This is the analogue of non-normalizable fall-offs in AdS. These fall-off behaviors are fixed by conducting the asymptotic (future/past) symmetry analysis in a manner closely parallel to the $\mathrm{AdS}_{3}$ counterpart [33, 34] as was done in [31. By demanding that the asymptotic symmetries of this larger theory still contain the Virasoro algebras already present in the $S L(2, C)$ case, it was found that the suitable fall-offs behaviors at future infinity for the $S L(3, C)$ gauge connections are

$$
A_{\bar{w}}=0, \quad A_{\rho}=b^{-1} \partial_{\rho} b, \quad A-A_{d S_{3}} \stackrel{\tau \rightarrow \infty}{\longrightarrow} \mathcal{O}(1) .
$$


Here $b$ is a gauge transformation $\in S L(3, C)$. However as we shall see in the next section, in order to construct gauge field configurations with non-vanishing higher spin charges, one has to violate the asymptotic fall-offs (8) and hence one has to supplement the action (1) with boundary terms. Again this is parallel to the situation for higher spin AdS black hole solutions [29] for which the boundary counter terms were worked out in [35, 36].

\section{Higher Spin de Sitter Cosmologies}

We are interested in constructing solutions of the $S L(3, C)$ gauge theory describing spacetimes of positive cosmological constant which have non-zero spin-3 charges in addition to the spin- 2 charges i.e. energy and angular momentum. Since these are higher spin extensions of the pure gravity solutions or $S L(2)$ sector, let us first review the solutions of the $S L(2)$ sector obtained by taking quotients of pure three-dimensional de Sitter space [8].

\section{1 $\quad$ Kerr-dS $_{3}$ universe}

The first class of $S L(2)$ quotients of pure de Sitter space is the so called Kerr - deSitter universe $\left(K d S_{3}\right)$. These are very similar to de Sitter space itself, in the sense that these solutions have two regions bounded by cosmological horizons, and have future and past infinite regions outside the cosmological horizons. However the topology of the past and future infinities of $K d S_{3}$ is that of a cylinder, $S^{1} \times R$, in contrast to the de Sitter space, for which they have topology of a sphere, $S^{2}$.

In static Schwarzschild-like coordinates, the $K d S_{3}$ metric [7, 8] reads like,

$$
d s^{2}=-N^{2}(r) d t^{2}+N^{-2}(r) d r^{2}+r^{2}\left(d \phi+N_{\phi} d t\right)^{2}, N^{2}(r)=M-\frac{r^{2}}{l^{2}}+\frac{J^{2}}{4 r^{2}}, N_{\phi}=-\frac{J}{2 r^{2}} .
$$

Introducing, the outer and inner radii

$$
r_{ \pm}^{2}=M l^{2}\left(\sqrt{1+(J / M l)^{2}} \pm 1\right) / 2
$$

one can rewrite Eq.(9) as

$$
d s^{2}=-\frac{\left(r^{2}+r_{-}^{2}\right)\left(r_{+}^{2}-r^{2}\right)}{r^{2} l^{2}} d t^{2}+\frac{r^{2} l^{2}}{\left(r^{2}+r_{-}^{2}\right)\left(r_{+}^{2}-r^{2}\right)} d r^{2}+r^{2}\left(d \phi+\frac{r_{+} r_{-}}{r^{2}} \frac{d t}{l}\right)^{2}, r<r_{+}
$$

and we note that this geometry has a horizon at $r=r_{+}$. This metric can be analytically continued across outside i.e. for $r>r_{+}$:

$$
d s^{2}=-\frac{r^{2} l^{2}}{\left(r^{2}+r_{-}^{2}\right)\left(r^{2}-r_{+}^{2}\right)} d r^{2}+\frac{\left(r^{2}+r_{-}^{2}\right)\left(r^{2}-r_{+}^{2}\right)}{r^{2} l^{2}} d t^{2}+r^{2}\left(d \phi+\frac{r_{+} r_{-}}{r^{2}} \frac{d t}{l}\right)^{2} .
$$

In this region $r$ is timelike while $t$ is spacelike. 
To make contact with the gauge theory we write down the $S L(2, C)$ connections for the two regions. Introducing, $\mathcal{N}^{2}(r) \equiv \frac{\left(r^{2}+r_{-}^{2}\right)\left(r^{2}-r_{+}^{2}\right)}{r^{2} l^{2}}=-N^{2}(r)$, the gauge field expressions are,

$$
\begin{aligned}
& A^{0}=N(r)\left(d \phi+i \frac{d t}{l}\right), \quad A^{1}=\frac{l N_{\phi}-i}{N(r)} \frac{d r}{l} \quad, A^{2}=\left(r N_{\phi}+i \frac{r}{l}\right)\left(d \phi+i \frac{d t}{l}\right) ; \quad r<r_{+}, \\
& A^{0}=-\frac{l N_{\phi}-i}{\mathcal{N}(r)} \frac{d r}{l}, \quad A^{1}=\mathcal{N}(r)\left(d \phi+i \frac{d t}{l}\right), \quad A^{2}=\left(r N_{\phi}+i \frac{r}{l}\right)\left(d \phi+i \frac{d t}{l}\right) ; \quad r>(\mathbb{1} 3)
\end{aligned}
$$

In the exterior region, $r>r_{+}^{2}$, on can transform to Fefferman-Graham like coordinates $(\rho, w, \bar{w})$ defined by

$$
\rho=\ln \left(\frac{\sqrt{r^{2}-r_{+}^{2}}+\sqrt{r^{2}+r_{-}^{2}}}{2 l}\right), w=\phi+i t / l, \bar{w}=\phi-i t / l
$$

and obtain the form of the metric,

$$
d s^{2}=-l^{2} d \rho^{2}+\frac{1}{2}\left(L d w^{2}+\bar{L} d \bar{w}^{2}\right)+\left(l^{2} e^{2 \rho}+\frac{L \bar{L}}{4} e^{-2 \rho}\right) d w d \bar{w}
$$

where, the zero modes, $L, \bar{L}$ are defined by,

$$
L+\bar{L}=M l, \quad L-\bar{L}=i J
$$

Note that $\rho$ here is a time coordinate, eg. [37.

This coordinate system is better suited than the Schwarzschild one for conducting the asymptotic symmetry analysis of $\mathrm{dS}_{3}$ and its identification with Euclidean Virasoro algebra and its charges [8]. As we have worked out in our previous paper [28], the corresponding $S L(2, C)$ gauge field is,

$$
A=i T_{0} d \rho+\left[\left(e^{\rho}-\frac{L}{2 l} e^{-\rho}\right) T_{1}+i\left(e^{\rho}+\frac{L}{2 l} e^{-\rho}\right) T_{2}\right] d w
$$

One can obtain the above Kerr- $\mathrm{dS}_{3}$ connection from a primitive connection, $a$ given by

$$
a=\left[\left(1-\frac{L}{2 l}\right) T_{1}+i\left(1+\frac{L}{2 l}\right) T_{2}\right] d w
$$

free of any $\rho$ dependence, by performing a single valued gauge transformation on $a$ :

$$
A=\mathcal{B}^{-1} a \mathcal{B}+\mathcal{B}^{-1} d \mathcal{B}
$$

for

$$
\mathcal{B}=\exp \left(i \rho T_{0}\right)=\exp \left(\rho L_{0}\right)
$$

(because $\mathcal{B}$ being a sole function of $\rho$ is single valued in the $\phi$ direction). 


\subsection{Quotient Cosmology}

One can also construct de Sitter quotients containing (spinning) big bang/big crunch singularities [8] (also reviewed in [38] ). These quotients are locally given by the same exterior Kerr- de Sitter metric (12). But since $t$ and $r$ switch their roles and become spacelike and timelike respectively, we are better off switching their roles in the metric itself,

$$
d s^{2}=-\frac{t^{2} l^{2}}{\left(t^{2}+r_{-}^{2}\right)\left(t^{2}-r_{+}^{2}\right)} d t^{2}+\frac{\left(t^{2}+r_{-}^{2}\right)\left(t^{2}-r_{+}^{2}\right)}{t^{2} l^{2}} d r^{2}+t^{2}\left(d \phi+\frac{r_{+} r_{-}}{t^{2}} d r\right)^{2} .
$$

The quotient cosmology arises when we compactify $r$ into a circle. With, $r$ and $\phi$ both being periodic the future and past infinity of this quotient cosmology have the topology of a torus, $S^{1} \times S^{1}$ as opposed to $R \times S^{1}$ for the case of the Kerr de Sitter universe. Also with a periodic $r$, this metric cannot be extended to $-r_{+}<t<r_{+}$where $g_{r r}<0$ and one has closed timelike curves. Removing this region then leaves us with a big bang (big crunch) like solution for $t>r_{+}\left(t<r_{+}\right)$ with the $r-\phi$ torus degenerating to a circle [8]. This is an example of a causal structure singularity [2], and these are the analogues of higher dimensional curvature singularities in $2+1$ dimensions.

These singularities were shown to be removable via a higher spin gauge transformation when we embed this metric into a spin-3 $S L(3)$ theory in [28].

Since the quotient cosmology is metrically identical to the exterior regions of the Kerr de Sitter universe, the Fefferman-Graham gauge metric expression (15) and the gauge connection expressions (171820) also carry over with the coordinate changes,

$$
\rho=\ln \left(\frac{\sqrt{t^{2}-r_{+}^{2}}+\sqrt{t^{2}+r_{-}^{2}}}{2 l}\right), w=\phi+i r / l, \bar{w}=\phi-i r / l .
$$

\subsection{The Higher Spin cosmological gauge fields}

In the $S L(3)$ theory, the general primitive connection that satisfies asymptotic (future) de Sitter fall off conditions is

$$
a^{\prime}=\left[\left(1-\frac{L}{2 l}\right) T_{1}+i\left(1+\frac{L}{2 l}\right) T_{2}+\frac{W}{8 l} W_{-2}\right] d w
$$

$L$ and $W$ can be functions of $z$, but we will consider the constant case in analogy with [29]. Explicit forms for the generators can be found in [28].

We can transform from the primitive connection $a^{\prime}$, to $A^{\prime}$, the fully $\rho$-dependent form by applying the transformation (20)

$$
A^{\prime}=i T_{0} d \rho+\left[\left(e^{\rho}-\frac{L}{2 l} e^{-\rho}\right) T_{1}+i\left(e^{\rho}+\frac{L}{2 l} e^{-\rho}\right) T_{2}+\frac{W}{8 l} e^{-2 \rho} W_{-2}\right] d w,
$$

which we call the Fefferman-Graham gauge because it manifests the proper $\rho \rightarrow \infty$ fall-offs behaviors Eq. (8) as derived in [33, 31]. 
As the trace of $W_{-2}$ with any $S L(3)$ generator is zero, we find that metric obtained from $A^{\prime}, \bar{A}^{\prime}$ is same as (15). But the spin-3 field now attains a non-zero value. These non-vanishing components of spin-3 fields are given by

$$
\begin{aligned}
\varphi_{w w w} & =-\frac{i}{8} l^{2} W \\
\varphi_{w w \bar{w}} & =-\frac{i}{24} l \bar{L} W e^{-2 \rho}+\frac{i}{24} l^{2} \bar{W}, \\
\varphi_{w \bar{w} \bar{w}} & =-\frac{i}{96} \bar{L}^{2} W e^{-4 \rho}+\frac{i}{24} l \bar{L} \bar{W} e^{-2 \rho}, \\
\varphi_{\bar{w} \bar{w} \bar{w}} & =\frac{i}{32} \bar{L}^{2} \bar{W} e^{-4 \rho} .
\end{aligned}
$$

In order to construct metrics (cosmologies) with nonvanishing spin-3 charges (which will necessarily violate the asymptotically $\mathrm{dS}$ fall-offs), we propose the following ansatz for the primitive connection corresponding to a general spin-3 cosmology

$$
a^{\prime}=\left[\left(1-\frac{L}{2 l}\right) T_{1}+i\left(1+\frac{L}{2 l}\right) T_{2}+\frac{W}{8 l} W_{-2}\right] d w+\mu\left[W_{2}+w_{0} W_{0}+w_{-2} W_{-2}+t\left(T_{1}-i T_{2}\right)\right] d \bar{w}
$$

where $\mu, w_{0}, w_{-2}$, and $t$ are constants. The motivation for this comes from the fact that under a suitable set of analytically continuations of the charges and sign of the cosmological constant (which will be elaborated in the following sections) de Sitter higher spin cosmologies turn into the Euclidean sections of AdS higher spin black hole solutions of [29] (much like in the case of pure gravity or $S L(2)$ sector, Kerr-dS $\mathrm{S}_{3}$ solutions continue on to Euclidean $B T Z$ black holes).

Now, the connection (26) is an off-shell object and contains too many independent parameters. Restricting on-shell, we find that the connection has to be of the form

$a^{\prime}=\left[\left(1-\frac{L}{2 l}\right) T_{1}+i\left(1+\frac{L}{2 l}\right) T_{2}+\frac{W}{8 l} W_{-2}\right] d w+\mu\left[W_{2}-\frac{L}{2 l} W_{0}+\frac{L^{2}}{16 l^{2}} W_{-2}+\frac{W}{l}\left(T_{1}-i T_{2}\right)\right] d \bar{w}$.

Now although the connection is on-shell, it is still arbitrary in the sense that one does not know whether such solutions make a regular or singular contribution to the Hartle-Hawking wavefunction (or equivalently, when continued to Euclidean AdS, the corresponding Gibbons-Hawking partition function, $\left.Z_{E C F T}\right)$. Just as in the second-order or metric formulation of gravity, this is guaranteed by demanding the regularity of the Euclidean section of the metric, in case of the first-order or connection formulation, it is fixed by demanding triviality of the gauge-connection $A$ along contractible circle(s). The non-trivial topology of the connection is captured by the holonomy matrix or the Wilson loop operator along any contractible circle, $\mathcal{C}$

$$
\operatorname{Hol}_{\mathcal{C}}(A) \equiv \mathcal{B}^{-1} \exp \left[\oint_{\mathcal{C}} d x^{\mu} a_{\mu}\right] \mathcal{B}=e^{H_{\mathcal{C}}}
$$


The triviality of the connection is ensured when this holonomy matrix is identity. Equivalently, this means that the matrix, $H_{\mathcal{C}}$ has eigenvalues $(0,-2 \pi i, 2 \pi i)$. In the case of Kerr- $\mathrm{dS}_{3}$ and its spin-3 generalizations one has a contractible thermo-angular circle,

$$
(t, \phi) \sim(t+i \beta, \phi+i \beta \Omega) .
$$

Equivalently, if one defines $\tau=\frac{\beta}{2 \pi}(1-i \Omega l)$, this thermo-angular circle can be reexpressed as $(w, \bar{w}) \sim(w+2 \pi \tau / l, \bar{w}+2 \pi \bar{\tau} / l)$.

The associated holonomy,

$$
\begin{aligned}
\operatorname{Hol}(a) & =\mathcal{B}^{-1} \exp \left[\int_{0}^{i \beta} d t a_{t}+\int_{0}^{i \beta \Omega} d \phi a_{\phi}\right] \mathcal{B} \\
& =\mathcal{B}^{-1} \exp \left[\int_{0}^{i \beta} d t i\left(a_{w}-a_{\bar{w}}\right) / l+\int_{0}^{i \beta \Omega} d \phi\left(a_{w}+a_{\bar{w}}\right)\right] \mathcal{B} \\
& =\mathcal{B}^{-1} \exp \left[-\left(2 \pi \tau a_{w}-2 \pi \bar{\tau} a_{\bar{w}}\right) / l\right] \mathcal{B} \\
& =e^{W(a)} .
\end{aligned}
$$

This means that the matrix $W(a)$ should have eigenvalues $(0,-2 \pi i, 2 \pi i) 6$. These two (complex) conditions entirely fix the charges $L, W$ in terms of the potentials $\beta, \mu$.

For generic gauge connections it is nontrivial to compute the holonomy matrix exactly. Since all we need are its eigenvalues, we are perfectly fine to work with the matrix, $\exp (\tilde{w}(a)), \tilde{w}(a) \equiv$ $-\left(2 \pi \tau a_{w}-2 \pi \bar{\tau} a_{\bar{w}}\right)$, instead, since it is related to the Holonomy matrix, $\exp (W(a))$ by a singlevalued gauge transformation (similarity transformation), $\mathcal{B}$ and hence has the same eigenvalue spectrum. Demanding the eigenvalues of the $\tilde{w}_{z}$ be $(0,-2 \pi i, 2 \pi i)$ implies

$$
\operatorname{det}(\tilde{w}(a))=0, \quad \operatorname{Tr}\left[\tilde{w}(a)^{2}\right]=-8 \pi^{2}, \quad \operatorname{Tr}[\tilde{w}(a)]=0 .
$$

which translate to the following relations determining the charges $L, W$ in terms of the potentials $\tau, \mu$,

$$
\begin{aligned}
27 l^{2} \tau^{3} W-36 l \tau^{2} \alpha L^{2}-54 l \tau \alpha^{2} L W-54 l \alpha^{3} W^{2}-8 \alpha^{3} L^{3} & =0 \\
1-\frac{2 \tau^{2} L}{l^{3}}-\frac{6 \tau \alpha W}{l^{3}}+\frac{4}{3} \frac{\alpha^{2} L^{2}}{\tau^{2} l^{2}} & =0
\end{aligned}
$$

where $\alpha=\mu \bar{\tau}$.

\footnotetext{
${ }^{6}$ Of course, one could consider the eigenvalues to be integer multiples of $\pm 2 \pi i$, in general, to get trivial holonomy. This ambiguity is directly tied to the ambiguity in identifying the period of the thermal circle with the inverse temperature, which in turn is ultimately tied to fixing the asymptotics of the geometry 39. The choice $(0,-2 \pi i, 2 \pi i)$ can be found in section 5.4 of $[29$. It is easy to see from (30) that once we fix a period $\tau$, scaling the holonomy eigenvalues by $N$ can only be accomplished by scaling the primitive connection $a$ in (27). But this results in a metric (and connection $A$ ) that will violate the standard Brown-Henneaux fall-offs (and their higher spin generalizations). This is again ultimately tied to the fact that the asymptotic fall-offs are defined after fixing the asymptotic coordinates; the Killing vectors are normalized at infinity. Eg: note that the norm of the Killing vector that turns null at the horizon is crucial for determining the surface gravity/temperature [39].
} 
Since we are already familiar with the exact solution for purely spin-2 charges i.e. mass and angular momentum, we can now obtain a solution to the charges in the presence of spin-3 potentials in a perturbation series in the spin-3 chemical potential, $\mu$ :

$$
W=\sum_{i=1}^{\infty} a_{i} \mu^{i} \quad L=\frac{l}{2 \tau^{2}}+\sum_{j=1}^{\infty} b_{j} \mu^{j}
$$

Substituting this in Eq.(32) and solving both equations to quadratic order $\mu$, we get following perturbative solution for $L$ and $W$

$$
\frac{L}{l}=\frac{l^{2}}{2 \tau^{2}}-\frac{5 \alpha^{2} l^{4}}{6 \tau^{6}}+\cdots, \quad \frac{W}{l}=\frac{\alpha l^{4}}{3 \tau^{5}}-\frac{20 \alpha^{3} l^{6}}{27 \tau^{9}}+\cdots
$$

These solutions satisfy the "integrability conditions" (as can be checked order by order),

$$
\frac{\partial L}{\partial \alpha}=\frac{\partial W}{\partial \tau}
$$

pointing out to the existence of a bulk (Euclidean) action, $I$

$$
\delta I \sim \delta \tau L+\delta \alpha W
$$

with $L$ and $W$ being functions of $\tau, \alpha$. The basic reason why eqn (35) arises is because we are demanding that there be an underlying partition function description for the system (the exponential of the action being the semi-classical partition function). The integrability condition is the statement that the double derivatives of the partition function (with respect to $\alpha$ and $\tau$ ) commute. A closely related discussion can be found in section (5.2) of [29]. The precise form of the action functional requires taking care of various subtleties, see [40]. We will make use of their results when we make comparisons with the AdS case.

For the (higher spin) AdS case, the integrability conditions were understood [29] to be integrability conditions of a boundary CFT partition function, $Z_{C F T}$ dual to the higher spin AdS bulk theory (vide AdS/CFT). The on-shell bulk action, $I^{\text {on-shell }}$ is the saddle-point contribution to $Z_{C F T}$, corresponding to the classical higher spin Black hole configuration. Similarly it will be shown in Sec. 4, the integrability conditions Eq. (32,34) for the case of (higher spin) de Sitter connections apply to that a putative dual Euclidean CFT partition function, $Z_{C F T *}$. It will also be shown that two partition functions $\left(Z_{C F T}, Z_{C F T *}\right)$ are related by a suitable "Wick-rotation" of the Cherns-Simons level number (cosmological constant) and gauge theory charges (mass, spin, spin-3 charges).

Finally we apply the radial gauge transformation, (20) to obtain full radial dependence,

$$
\begin{aligned}
& A^{\prime} \quad=i T_{0} d \rho+\left[\left(e^{\rho}-e^{-\rho} \frac{L}{2 l}\right) T_{1}+i\left(e^{\rho}+e^{-\rho} \frac{L}{2 l}\right) T_{2}+e^{-2 \rho} \frac{W}{8 l} W_{-2}\right] d w \\
& +\mu\left[e^{2 \rho} W_{2}-\frac{L}{2 l} W_{0}+e^{-2 \rho} \frac{L^{2}}{16 l^{2}} W_{-2}+e^{-\rho} \frac{W}{l}\left(T_{1}-i T_{2}\right)\right] d \bar{w}, \\
& \bar{A}^{\prime}=-i T_{0} d \rho+\left[\left(e^{\rho}-e^{-\rho} \frac{\bar{L}}{2 l}\right) T_{1}-i\left(e^{\rho}+e^{-\rho} \frac{\bar{L}}{2 l}\right) T_{2}+e^{-2 \rho} \frac{\bar{W}}{8 l} W_{-2}\right] d \bar{w} \\
& +\bar{\mu}\left[e^{2 \rho} W_{2}-\frac{L}{2 l} W_{0}+e^{-2 \rho} \frac{L^{2}}{16 l^{2}} W_{-2}+e^{-\rho} \frac{\bar{W}}{l}\left(T_{1}+i T_{2}\right)\right] d w .
\end{aligned}
$$


The corresponding metric expression is

$$
\begin{aligned}
d s^{2}= & -l^{2} d \rho^{2}+\left(\frac{l L}{2}+\frac{l W \bar{\mu}}{2}-\frac{1}{2} e^{-2 \rho} L \bar{W} \bar{\mu}-\frac{\bar{L}^{2} \bar{\mu}^{2}}{3}\right) d w^{2}+\left(\frac{l \bar{L}}{2}+\frac{l \bar{W} \mu}{2}-\frac{1}{2} e^{-2 \rho} \bar{L} W \mu-\frac{L^{2} \mu^{2}}{3}\right) d \bar{w}^{2} \\
& \left(\frac{1}{2} e^{2 \rho} l^{2}-\frac{3 l W \mu}{4}-\frac{3 l \bar{W} \bar{\mu}}{4}+\frac{L^{2} \mu \bar{\mu}}{8}+\frac{L \bar{L} \mu \bar{\mu}}{12}+\frac{\bar{L}^{2} \mu \bar{\mu}}{8}+\frac{1}{8} e^{-2 \rho}(L \bar{L}+4 W \bar{W} \mu \bar{\mu})\right) d w d \bar{w} .
\end{aligned}
$$

while the expressions for the non-vanishing spin-3 field components are,

$$
\begin{aligned}
& \psi_{\rho \rho w}=\frac{1}{18} i l^{2} \bar{L} \bar{\mu}, \quad \quad \psi_{\rho \rho \bar{w}}=-\frac{1}{18} i l^{2} L \mu, \\
& \psi_{w w w}=-\frac{1}{8} i l^{2} W+\frac{1}{16} i l L^{2} \bar{\mu}+\frac{1}{24} i l L \bar{L} \bar{\mu}+\frac{1}{16} i l \bar{L}^{2} \bar{\mu}-\frac{1}{12} i l \bar{L} W \bar{\mu}^{2}+\frac{1}{27} i \bar{L}^{3} \bar{\mu}^{3} \\
& +\frac{1}{4} i e^{-2 \rho}\left(l W \bar{W} \bar{\mu}-\frac{1}{6} L \bar{L} \bar{W} \bar{\mu}-\frac{1}{2} \bar{L}^{2} \bar{W} \bar{\mu}^{2}\right)-\frac{1}{8} i e^{-4 \rho} W \bar{W}^{2} \bar{\mu}^{2}+\frac{i e^{-4 \rho} \bar{L}^{2} \bar{W}^{2} \bar{\mu}^{3}}{16 l} . \\
& \psi_{\bar{w} \bar{w} \bar{w}}=-\frac{1}{4} i e^{4 \rho} l^{3} \mu+\frac{1}{2} i e^{2 \rho} l^{2} W \mu^{2}-\frac{1}{24} i l L \bar{L} \mu+\frac{1}{12} i l L \bar{W} \mu^{2}-\frac{1}{27} i L^{3} \mu^{3}-\frac{1}{4} i l W^{2} \mu \\
& +\frac{1}{24} i e^{-2 \rho} L \bar{L} W \mu^{2}+\frac{1}{32} i e^{-4 \rho}\left(\bar{L}^{2} \bar{W}-\frac{L^{2} \bar{L}^{2} \mu}{2 l}\right), \\
& \psi_{w w \bar{w}}=\frac{1}{12} i e^{2 \rho}\left(l^{2} L \bar{\mu}+\frac{1}{3} i l^{2} \bar{L} \bar{\mu}\right) \\
& +\frac{1}{24} i l^{2} \bar{W}-\frac{1}{18} i l L^{2} \mu-\frac{1}{18} i l L W \mu \bar{\mu}-\frac{1}{72} i L^{2} \bar{L} \mu \bar{\mu}^{2}-\frac{1}{108} i L \bar{L}^{2} \mu \bar{\mu}^{2}-\frac{1}{72} i \bar{L}^{3} \mu \bar{\mu}^{2} \\
& +\frac{i e^{-2 \rho}}{12}\left(\frac{1}{12} L \bar{L}^{2} \bar{\mu}+\frac{1}{4} \bar{L}^{3} \bar{\mu}-l \bar{W}^{2} \bar{\mu}-\frac{1}{2} l \bar{L} W+\frac{2}{3} L^{2} \bar{W} \mu \bar{\mu}+\frac{1}{36} \bar{L} W \bar{W} \mu \bar{\mu}^{2}\right) \\
& -\frac{i e^{-4 \rho}}{24}\left(\frac{\bar{L}^{3} \bar{W} \bar{\mu}^{2}}{2 l}-\bar{L} W \bar{W} \bar{\mu}-\bar{W}^{3} \bar{\mu}^{2}+\frac{L^{2} \bar{W}^{2} \mu \bar{\mu}^{2}}{2 l}\right), \\
& \psi_{w w \bar{w}}=\frac{1}{12} i e^{4 \rho} l^{3} \bar{\mu}-i e^{2 \rho} l^{2}\left(\frac{1}{9} L \mu+\frac{1}{6} W \mu \bar{\mu}\right) \\
& +\frac{1}{12} i\left(l L W \mu^{2}+\frac{1}{6} l \bar{L}^{2} \bar{\mu}-\frac{1}{3} l \bar{L} \bar{W} \mu \bar{\mu}+\frac{1}{6} L^{3} \mu^{2} \bar{\mu}+\frac{1}{9} L^{2} \bar{L} \mu^{2} \bar{\mu}+\frac{1}{6} L \bar{L}^{2} \mu^{2} \bar{\mu}+l W^{2} \mu^{2} \bar{\mu}\right) \\
& +\frac{1}{12} i e^{-2 \rho}\left(l \bar{L} \bar{W}-\frac{1}{3} L^{2} \bar{L} \mu-\frac{1}{6} \bar{L}^{2} W \mu \bar{\mu}-\frac{1}{3} L W \bar{W} \mu^{2} \bar{\mu}\right) \\
& -\frac{1}{24} i e^{-4 \rho}\left(\bar{L}^{2} W-\frac{\bar{L}^{4} \bar{\mu}}{8 l}+\bar{L} \bar{W}^{2} \bar{\mu}-\frac{L^{2} \bar{L} \bar{W} \mu \bar{\mu}}{2 l}\right)(
\end{aligned}
$$

\subsection{Schwarzschild Gauge}

The Fefferman-Graham (FG) gauge expressions only cover a part of the spacetime outside the horizon. In this section, we describe solution of the gauge connection in Schwarzschild gauge. For 
simplicity, we will consider the purely non-rotating case from now on,

$$
L=\bar{L}, \quad \bar{W}=-W \quad \text { and } \quad \bar{\mu}=-\mu .
$$

The metric in FG gauge is then,

$$
\begin{aligned}
g_{\rho \rho} & =-l^{2}, \\
g_{t t} & =\left(e^{\rho}-\frac{L+2 W \mu}{2 l} e^{-\rho}\right)^{2}, \\
g_{\phi \phi} & =l^{2}\left(e^{\rho}+\frac{L-2 W \mu}{2 l} e^{-\rho}\right)^{2}+\frac{4 L^{2}|\mu|^{2}}{3}-2 l W \mu .
\end{aligned}
$$

We observe that there is a horizon i.e. $g_{t t}$ vanishes, at

$$
\rho_{+}=\frac{1}{2} \ln \left[\frac{L+2 W \mu}{2 l}\right] .
$$

Now, we can introduce the Schwarzschild radial coordinate $r$ (motivated from the definition of the Schwarzschild radial coordinate for the pure $S L(2)$ case),

$$
\rho=\ln \left[\frac{r+\sqrt{r^{2}-r_{+}^{2}}}{2 l}\right]
$$

where $r_{+}^{2}$ is

$$
r_{+}^{2}=2 l(L+2 W \mu) .
$$

In the limit $\mu=0$, the above equation reduces to the pure $S L(2)$ case, (14). In the Schwarzschild like gauge the metric is given by,

$$
\begin{aligned}
d s^{2}= & -\frac{l^{2}}{r^{2}-r_{+}^{2}} d r^{2}+\frac{2(L+2 W \mu)}{l r_{+}^{2}}\left(r^{2}-r_{+}^{2}\right) d t^{2}+ \\
& {\left[\left(\frac{r L}{L+2 W \mu}+\frac{2 l \mu W \sqrt{r^{2}-r_{+}^{2}}}{L+2 W \mu}\right)^{2}+\frac{4 L^{2}|\mu|^{2}}{3}-2 l W \mu\right] d \phi^{2} }
\end{aligned}
$$

We also note that $g_{\phi \phi}>0$ as it is a sum of manifestly positive quantities ( $W$ and $\mu$ are imaginary quantities with same sign vide (34)) and there are no closed timelike curves in the $\phi$ direction.

\subsection{Higher Spin Quotient Cosmologies}

Now that we have the metric expressions for the higher spin versions of the Kerr de Sitter universe in Schwarzschild gauge (45) outside the cosmological horizon, one can now write down metric for higher spin generalizations of the quotient cosmologies (21) by simply swapping $r$ and $t$.

$d s^{2}=-\frac{l^{2}}{t^{2}-r_{+}^{2}} d t^{2}+\frac{2(L+2 W \mu)}{l r_{+}^{2}}\left(t^{2}-r_{+}^{2}\right) d r^{2}+\left[\left(\frac{L t+2 \mu W \sqrt{t^{2}-r_{+}^{2}}}{L+2 W \mu}\right)^{2}+\frac{4 L^{2}|\mu|^{2}}{3}-2 l W \mu\right] d \phi^{2}$. 
Just as in the case for the $S L(2)$ quotient cosmology, $r$ is now compactified into a circle and this metric cannot be continued inside the horizon, $r_{+}$. As a result this it contains big bang/big crunch like singularities at $t= \pm r_{+}$when the $r$-circle degenerates to a point, exactly like its $S L(2)$ cousin. It will be interesting to consider the resolution of these singularities along the lines of [28, but we will not pursue it here.

\section{Thermodynamics of asymptotically de Sitter connections}

The aim of this section to derive a consistent thermodynamics for asymptotically $\mathrm{dS}_{3}$ spin- 2 connections in the Chern-Simons language. (See [43] for an explicit expression for the entropy in metric-like variables.) In a metric (second order) formalism of gravity, more precisely spin-2 gravity, thermodynamics of spacetimes containing horizons of any kind is provided by the GibbonsHawking generalization [41] of the black hole thermodynamics of Bardeen, Carter and Hawking 42. However, as we shall see, in the Chern-Simons or first order set-up, a consistent thermodynamics is obtained extremely efficiently by first mapping de Sitter solutions to Euclidean AdS (EAdS) solutions and then demanding integrability conditions on free energy (equivalently partition function) of a putative Euclidean CFT located on the future infinity of the asymptotic de Sitter (same as conformal boundary of the analytically continued EAdS solution). Maldacena [12] notes that the conformal patch of $\mathrm{dS} 7$,

$$
d s^{2}=\frac{-d \eta^{2}+d \mathbf{x}_{d}^{2}}{\eta^{2} / l^{2}}
$$

goes over to the Poincare patch of the EAdS, under, $l^{2} \rightarrow-l^{2}$ and, $\eta^{2} \rightarrow-z^{2}$,

$$
d s^{2}=\frac{d z^{2}+d \mathbf{x}_{d}^{2}}{z^{2} / l^{2}},
$$

and then he proposes that for any asymptotic (in time) de Sitter space,

$$
\Psi_{\text {Hartle-Hawking }}=Z_{C F T^{*}}
$$

since for EAdS one has the celebrated AdS-CFT conjecture $Z_{E S U G R A}=Z_{C F T}$. Under the identifications, the Euclidean path-integral in AdS becomes the Hartle-Hawking wave function of dS. Next we construct a similar map between the exterior regions of Kerr deSitter and and Euclidean BTZ black hole and then generalize to the higher spin case where the bulk action would be a first order action instead of second order (metric) action.

\footnotetext{
${ }^{7}$ ie., the upper quadrant in the dS Penrose diagram containing the infinite future at $\eta=0$ and bounded by the horizon at $\eta=1$. No light rays from the infinite past can reach this region.
} 


\section{1 "Wick-rotation" from Kerr de Sitter to $E B T Z$}

We simply write down these identifications for the FG gauge,

$$
\begin{aligned}
\rho_{d S} & \rightarrow \rho_{E A d S}+i \frac{\pi}{2} \\
t_{d s} & \rightarrow i t_{E A d S} \\
l_{d S} & \rightarrow i l_{E A d S}, \\
L_{d S}, \bar{L}_{d S} & \rightarrow-i L_{E A d S},-i \bar{L}_{E A d S} \\
M_{d S}, J_{d S} & \rightarrow-M_{A d S},-J_{A d S} .
\end{aligned}
$$

Under these identifications, the $\mathrm{KdS}_{3}$ metric Eq. (12) goes over to

$$
d s^{2} \rightarrow d \tilde{s}^{2}=l^{2} d \rho^{2}+\frac{l}{2}\left(L d w^{2}+\bar{L} d \bar{w}^{2}\right)+\left(l^{2} e^{2 \rho}+\frac{L \bar{L}}{4} e^{-2 \rho}\right) d w d \bar{w}
$$

but now with,

$$
L=\frac{M l+J}{2}, \bar{L}=\frac{M l-J}{2} .
$$

This is an evidently an Euclidean metric. To determine whether this is the Euclidean $B T Z$ metric $(E B T Z)$, we write the $E B T Z$ metric expressions directly from Euclideanizing the Lorentzian $B T Z$,

$$
d s^{2}=\frac{l}{2}\left(L^{+} d w^{+2}+L^{-} d w^{-2}\right)+\left(l^{2} e^{2 \rho}+\frac{L^{+} L^{-}}{4} e^{-2 \rho}\right) d w^{+} d w^{-}+l^{2} d \rho^{2}, w^{ \pm}=\phi \pm \frac{t}{l},
$$

where, the "zero modes" $L^{+}, L^{-}$are defined in terms of the mass and the spin by,

$$
L^{+}=\frac{M l+J}{2}, L^{-}=\frac{M l-J}{2} .
$$

Upon a replacing $t \rightarrow i t_{E}$, we obtain the $E B T Z$ metric,

$$
d s^{2}=\frac{l}{2}\left(L^{+} d w^{2}+L^{-} d \bar{w}^{2}\right)+\left(l^{2} e^{2 \rho}+\frac{L^{+} L^{-}}{4} e^{-2 \rho}\right) d w d \bar{w}+l^{2} d \rho^{2}, w=\phi+\frac{i t_{E}}{l}, \bar{w}=\phi-\frac{i t_{E}}{l},
$$

Clearly, this is identical to the wick-rotated $K d S_{3}$ metric Eq. (52).

\subsubsection{Schwarzschild Gauge Wick-rotation}

In Schwarzschild-like coordinates, the Kerr-dS $\mathrm{S}_{3}$ metric 9, on using the identifications, 51, becomes,

$$
d \tilde{s}^{2}=N^{2} d t^{2}+N^{-2} d r^{2}+r^{2}\left(i N^{\phi} d t+d \phi\right)^{2}, N^{2}=-M+\frac{r^{2}}{l^{2}}+\frac{J^{2}}{4 r^{2}}, N^{\phi}=\frac{J}{2 r^{2}} .
$$

The Lorentzian exterior $B T Z$ metric Eq. (54) reads,

$$
d s^{2}=-N^{2} d t^{2}+N^{-2} d r^{2}+r^{2}\left(N^{\phi} d t+d \phi\right)^{2}, N^{2}=-M+\frac{r^{2}}{l^{2}}+\frac{J^{2}}{4 r^{2}}, N^{\phi}=\frac{J}{2 r^{2}} .
$$


which upon Euclideanizing i.e. $t \rightarrow i t_{E}$,

$$
d s^{2}=N_{E}^{2} d t_{E}^{2}+d r^{2} / N_{E}^{2}+r^{2}\left(i N_{E}^{\phi} d t_{E}+d \phi\right)^{2}, N_{E}^{2}=-M+\frac{r^{2}}{l^{2}}+\frac{J^{2}}{4 r^{2}}, N_{E}^{\phi}=\frac{J}{2 r^{2}} .
$$

One can write a metric expression in terms of outer and inner horizons for the $B T Z$ along the lines of 12 ,

$$
d s^{2}=-\frac{\left(r^{2}-r_{+}^{2}\right)\left(r^{2}-r_{-}^{2}\right)}{r^{2} l^{2}} d t^{2}+\frac{r^{2} l^{2}}{\left(r^{2}-r_{+}^{2}\right)\left(r^{2}-r_{-}^{2}\right)} d r^{2}+r^{2}\left(d \phi+\frac{r_{+} r_{-}}{r^{2}} \frac{d t}{l}\right)^{2}, r>r_{+}
$$

with,

$$
r_{ \pm}^{2}=\frac{M l^{2}}{2}\left(1 \pm \sqrt{1-\left(\frac{J}{M l}\right)^{2}}\right) .
$$

So the identifications are,

$$
r_{+} \rightarrow r_{+},\left(r_{-}\right)_{K d S_{3}} \rightarrow-i\left(r_{-}\right)_{B T Z} .
$$

For $K d S_{3}$ note that in terms of $L, \bar{L}$,

$$
\begin{gathered}
r_{+}=\frac{\sqrt{L l}+\sqrt{\bar{L} l}}{\sqrt{2}}, r_{-}=\frac{\sqrt{L l}+\sqrt{\overline{L l}}}{\sqrt{2}} \\
r_{+}^{2}+r_{-}^{2}=2 \sqrt{L \bar{L} l^{2}} .
\end{gathered}
$$

So, the temperature inverse of $K d S_{3}$ in terms of $L, \bar{L}$,

$$
\frac{\beta}{2 \pi}=\frac{l^{2}}{2}\left(\frac{1}{\sqrt{2 L l}}+\frac{1}{\sqrt{2 \overline{L l}}}\right) .
$$

For non-rotating $K d S_{3}, \tau=\beta / 2 \pi$ and we have

$$
\frac{L}{l}=\frac{l^{2}}{2 \tau^{2}} .
$$

Again, this metric (59) is exactly that of the Wick-rotated dS metric in Schwarzschild coordinates, Eq. (57).

\section{$4.2 \mathrm{dS}-\mathrm{AdS}$ "wick rotation" at work: Equivalence of thermodynamics in the metric formulation}

In order to further solidify our heuristic identifications, we show that under these identifications the Gibbons-Hawking thermodynamics [41], including the temperature and entropy of the Kerr-dS solution, maps onto to those the "wick-rotated" EBTZ solutions. 
1. The entropies for either geometry are the same since entropy of either cosmological or Blackhole horizons in the Gibbons-Hawking framework is given by

$$
S=\frac{1}{4 G}(\text { Horizon Area })=2\left(2 \pi r_{+}\right)=4 \pi r_{+}
$$

This is borne out by our heuristic identifications, since $r_{+} \rightarrow r_{+}$.

2. The temperature of $\mathrm{KdS}_{3}$ is given by Gibbons-Hawking thermodynamics by the conical singularity trick,

$$
T_{K d S_{3}}=\frac{r_{+}^{2}+r_{-}^{2}}{2 \pi l^{2} r_{+}}
$$

Using the identification Eq. (62) and the additional identification $T_{d s} \rightarrow-T_{A d S} 8$ this temperature continues to the Hawking temperature of the corresponding BTZ black hole!

$$
T_{B T Z}=\frac{r_{+}^{2}-r_{-}^{2}}{2 \pi l^{2} r_{+}} .
$$

3. The chemical potential conjugate to angular momentum is,

$$
\Omega_{K d S_{3}}=-T \frac{\partial S}{\partial J}=-\frac{r_{-}}{r_{+} l}
$$

Again under the identifications, we obtain the expected behavior $\Omega_{d S} \rightarrow \Omega_{A d S}$ since $J_{d S} \rightarrow$ $-J_{A d S} 9$. We note that, $\Omega_{B T Z}=\frac{r_{-}}{r_{+}}$. Parenthetically, we note that when we move to Euclidean BTZ, we need to define, $J_{E A d S}=-i J_{A d S}$ and consequently the new conjugate $\Omega_{E A d S}=i \Omega_{A d S}$, so that respective identifications are, $J_{d S} \rightarrow-i J_{E A d S}$ and $\Omega_{K d S_{3}} \rightarrow-i \Omega_{E B T Z}$.

${ }^{8}$ This temperature sign flip is a direct result of the flip in the sign of mass parameter or "internal energy" $M$ in identification 51, The conjugacy relation

$$
T^{-1}=\frac{\partial S}{\partial M}
$$

directs you that one needs to perform,

$$
T_{d S} \rightarrow-T_{A d S}
$$

in consonance with

$$
M_{d S} \rightarrow-M_{A d S}
$$

\footnotetext{
${ }^{9}$ Since, going over from dS to AdS implies the replacements $S \rightarrow S, J \rightarrow-J$ and $\beta \rightarrow-\beta$, we must have $\Omega \rightarrow \Omega$
} in order to reproduce the correct thermodynamic relation,

$$
\frac{\partial S}{\partial J}=-\Omega \beta
$$


Since under the identifications, one can successfully map any dS thermodynamic quantities like entropy, internal energy, angular charges and their respective conjugates to AdS quantities, the laws of thermodynamics will continue as well. When higher spin charges are added, we will demand a similar statement to hold with higher spin charges and chemical potential added to the thermodynamical relations.

\subsection{Thermodynamics in the Chern-Simons formulation}

So far everything we discussed was in the $S L(2, C)$ sector of the theory with just metric or spin-2 fields turned on, but we extend this analogy to the full $S L(3, C)$ sector i.e. when both metric and spin-3 field are present. In that case though we do not know the generalization of the GibbonsHawking thermodynamics [42]. However, taking $\mathrm{dS} / \mathrm{CFT}$ as a principle, we can propose that the thermodynamics of a dS-connection is identical to that of a suitably continued Euclidean AdS-connection i.e. a higher spin AdS black hole [29]. The thermodynamics of $S L(3, C)$ valued Euclidean $\mathrm{AdS}_{3}$-connections for higher spin black-holes (connected to BTZ, i.e. the so called "BTZ" branch) has been shown to be dictated by the integrability conditions of the free energy of a dual CFT [29]. These conditions which can be cast in a gauge-invariant form by the holonomy conditions [29]. Under the correct identifications of charges and potentials, the integrability or holonomy conditions of a dS connection should continue to those of an AdS connection. Or turning this fact around, we expect the charges we obtain functions of the potentials $\mu$ and $T$ on solving the integrability conditions on the dS side, (34) to reproduce the respective solution of AdS integrability conditions i.e. AdS charges as a function of AdS potentials [40] upon making the dS-to-AdS identifications. For AdS, the solution to the holonomy conditions is,

$$
\frac{L^{+}}{l}=\frac{l^{2}}{2 \tau^{2}}+\frac{10}{3} \frac{\alpha^{2} l^{4}}{\tau^{6}}+\ldots, \quad \frac{W^{+}}{l}=-\frac{4}{3} \frac{\alpha l^{4}}{\tau^{5}}+\ldots
$$

We have the identifications for the spin-3 charges when going from $d S$ to $A d S$,

$$
\alpha_{A d S}=\frac{\alpha_{d S}}{2}, W_{A d S}^{+}=-2 i W_{d S},
$$

or,

$$
\mu_{A d S}=-\frac{\mu_{d S}}{2}, W_{A d S}^{+}=-2 i W_{d S}
$$

\subsubsection{The Action and Free energy in the CS theory}

To compute, $Z_{C F T}$ from the bulk gauge theory, we make use of the saddle point approximation,

$$
Z_{C F T}=Z_{(E) S U G R A}=e^{I_{E}^{\text {On-shell }}}
$$

where, $I_{E}$ is the Euclidean bulk action, defined in terms of the original action, $I$ by

$$
I_{E}[F(\mathbf{x}, t)]=i I\left[F\left(\mathbf{x}, i t_{E}\right)\right]
$$


The Chern-Simons action without any supplementary boundary terms,

$$
I_{C S}=\frac{k}{4 \pi \epsilon_{R}} \int \operatorname{Tr}\left(A d A+\frac{2}{3} A^{3}\right), \epsilon_{R}=4,
$$

is the right action for the $S L(2)$ sector. On-shell this becomes [44],

$$
I_{C S}[A]=-\frac{k}{4 \pi \epsilon_{R}} \int d t d \phi \operatorname{Tr}\left(A_{t} A_{\phi}\right) .
$$

For $S L(3)$ sector one needs to add new boundary terms as formulated in [35, 36]. But it is easy to see that a similar map as we are presenting below will also hold for the boundary terms, so in the following, we will illustrate it only for the bulk terms. Using, (5.1) of [29]

$$
I_{\text {on-shell }}^{\mathrm{EAdS}}=-\frac{2 \beta L}{l}+\frac{16 \beta \mu^{2} L^{2}}{3 l^{2}}
$$

The higher spin de Sitter on-shell action turns out to be10,

$$
\tilde{I}_{\text {on-shell }}^{\mathrm{dS}}=-\left(\frac{2 \beta L}{l}+\frac{4}{3} \frac{\beta \mu^{2} L^{2}}{l^{2}}\right) .
$$

Again, using the identifications, we see that the $d S$ on-shell action reproduces the $E A d S$ on-shell action, (82)

$$
\tilde{I}_{\text {On-shell }}^{\mathrm{dS}}=I_{\text {On-shell }}^{\mathrm{EAdS}}
$$

Thus we have demonstrated that the higher spin generalizations of Kerr de Sitter universes are related to (higher spin) AdS black holes just as they were in the pure gravity (spin-2) case in the metric formulation. However this on-shell action is not yet equal to a $-\beta \Phi$, where $\Phi$ is the grand "higher spin" canonical potential $\Phi=E-T S-\mu W$. But it is possible to add boundary/supplementary terms and change the action $\tilde{I}_{\text {on-shell }}^{\mathrm{dS}}$ to a new action $I_{\text {On-shell }}$ such that,

$$
-I_{\text {On-shell }}=\beta \Phi \text {. }
$$

Such a procedure was conducted in the anti de Sitter case in [40], see their section (2.2). For our de Sitter case, the necessary extra terms can be obtained from their expressions by the AdS-dS identifications (51) and (77), in exact analogy with our computation here for the bulk terms. The match between our entropy and the higher spin $\mathrm{AdS}_{3}$ black hole entropy [45] is a natural consequence, and we have explicitly checked this. This concludes our discussion about the connection between the thermodynamics of the Kerr- $\mathrm{dS}_{3}$ solution and that of higher spin black holes in $\mathrm{AdS}_{3}$.

\footnotetext{
${ }^{10}$ To get this on-shell action, we perform integration over $t$-circle $(0, i \beta)$ and over $\phi$-circle $(0,2 \pi)$

$$
\tilde{I}_{\text {on-shell }}^{\mathrm{dS}}=i\left(-\frac{k}{4 \pi \epsilon_{R}}\right) \int_{0}^{-\beta}(i d t) \int_{0}^{2 \pi} d \phi\left[-i \operatorname{Tr}\left(A_{t} A_{\phi}-\bar{A}_{t} \bar{A}_{\phi}\right)\right],
$$
}

with

$$
k=-2 i l .
$$




\section{Acknowledgments}

We thank Justin David for clarifications on [40]. CK thanks Oleg Evnin for discussions on higher spins and singularity resolution, and Oleg Evnin and Auttakit Chatrabhuti for hospitality at Chulalangkorn University, Bangkok, during part of this project. The research of SR is supported by Department of Science and Technology (DST), Govt. of India research grant under scheme DSTO/1100 (ACAQFT).

\section{References}

[1] M. Banados, C. Teitelboim, and J. Zanelli, "The Black hole in three-dimensional space-time," Phys.Rev.Lett. 69 (1992) 1849-1851, hep-th/9204099.

[2] M. Banados, M. Henneaux, C. Teitelboim, and J. Zanelli, "Geometry of the (2+1) black hole," Phys.Rev. D48 (1993) 1506-1525, gr-qc/9302012.

[3] E. Witten, "(2+1)-Dimensional Gravity as an Exactly Soluble System," Nucl.Phys. B311 (1988) 46.

[4] A. Achucarro and P. Townsend, "A Chern-Simons Action for Three-Dimensional anti-De Sitter Supergravity Theories," Phys.Lett. B180 (1986) 89.

[5] J. D. Brown and M. Henneaux, "Central Charges in the Canonical Realization of Asymptotic Symmetries: An Example from Three-Dimensional Gravity," Commun.Math.Phys. 104 (1986) 207-226.

[6] J. M. Maldacena, "The Large N limit of superconformal field theories and supergravity," Adv.Theor.Math.Phys. 2 (1998) 231-252, hep-th/9711200.

[7] M.-I. Park, "Statistical entropy of three-dimensional Kerr-de Sitter space," Phys.Lett. B440 (1998) 275-282, hep-th/9806119.

[8] V. Balasubramanian, J. de Boer, and D. Minic, "Mass, entropy and holography in asymptotically de Sitter spaces," Phys.Rev. D65 (2002) 123508, hep-th/0110108.

[9] M. Spradlin, A. Strominger, and A. Volovich, "Les Houches lectures on de Sitter space," hep-th/0110007.

[10] E. Witten, "Quantum gravity in de Sitter space," hep-th/0106109.

[11] A. Strominger, "Inflation and the dS / CFT correspondence," JHEP 0111 (2001) 049, hep-th/0110087.

[12] J. M. Maldacena, "Non-Gaussian features of primordial fluctuations in single field inflationary models," JHEP 0305 (2003) 013, astro-ph/0210603.

[13] A. Polyakov, "De Sitter space and eternity," Nucl.Phys. B797 (2008) 199-217, 0709.2899. 
[14] T. Banks, "Some thoughts on the quantum theory of stable de Sitter space," hep-th/0503066.

[15] W. Fischler, A. Kashani-Poor, R. McNees, and S. Paban, "The Acceleration of the universe, a challenge for string theory," JHEP 0107 (2001) 003, hep-th/0104181.

[16] L. Dyson, J. Lindesay, and L. Susskind, "Is there really a de Sitter/CFT duality?," JHEP 0208 (2002) 045, hep-th/0202163.

[17] L. Dyson, M. Kleban, and L. Susskind, "Disturbing implications of a cosmological constant," JHEP 0210 (2002) 011, hep-th/0208013.

[18] C. Krishnan and E. di Napoli, "Can quantum de Sitter space have finite entropy?," Class.Quant.Grav. 24 (2007) 3457-3463, hep-th/0602002.

[19] B. Sundborg, "Stringy gravity, interacting tensionless strings and massless higher spins," Nucl.Phys.Proc.Suppl. 102 (2001) 113-119, hep-th/0103247.

[20] E. Fradkin and M. A. Vasiliev, "Candidate to the Role of Higher Spin Symmetry," Annals Phys. 177 (1987) 63.

[21] E. Fradkin and M. A. Vasiliev, "On the Gravitational Interaction of Massless Higher Spin Fields," Phys.Lett. B189 (1987) 89-95.

[22] M. A. Vasiliev, "Closed equations for interacting gauge fields of all spins," JETP Lett. 51 (1990) 503-507.

[23] M. A. Vasiliev, "Consistent equation for interacting gauge fields of all spins in (3+1)-dimensions," Phys.Lett. B243 (1990) 378-382.

[24] M. A. Vasiliev, "More on equations of motion for interacting massless fields of all spins in (3+1)-dimensions," Phys.Lett. B285 (1992) 225-234.

[25] M. A. Vasiliev, "Higher spin gauge theories: Star product and AdS space," hep-th/9910096.

[26] C. Fronsdal, "Massless Fields with Integer Spin," Phys.Rev. D18 (1978) 3624.

[27] M. Blencowe, "A CONSISTENT INTERACTING MASSLESS HIGHER SPIN FIELD THEORY IN D = (2+1)," Class.Quant.Grav. 6 (1989) 443.

[28] C. Krishnan and S. Roy, "Higher Spin Resolution of a Toy Big Bang," 1305.1277.

[29] M. Gutperle and P. Kraus, "Higher Spin Black Holes," JHEP 1105 (2011) 022, 1103.4304.

[30] M. Ammon, M. Gutperle, P. Kraus, and E. Perlmutter, "Spacetime Geometry in Higher Spin Gravity," JHEP 1110 (2011) 053, 1106.4788.

[31] P. Ouyang, "Toward Higher Spin dS3/CFT2," 1111.0276. 
[32] S. Lal and B. Sahoo, "Holographic Renormalisation for the Spin-3 Theory and the (A)dS3/CFT2 correspondence," JHEP 1301 (2013) 004, 1209.4804.

[33] A. Campoleoni, S. Fredenhagen, S. Pfenninger, and S. Theisen, "Asymptotic symmetries of three-dimensional gravity coupled to higher-spin fields," JHEP 1011 (2010) 007, 1008.4744.

[34] M. Henneaux and S.-J. Rey, "Nonlinear $W_{\text {infinity }}$ as Asymptotic Symmetry of Three-Dimensional Higher Spin Anti-de Sitter Gravity," JHEP 1012 (2010) 007, 1008.4579.

[35] M. Banados, R. Canto, and S. Theisen, "The Action for higher spin black holes in three dimensions," JHEP 1207 (2012) 147, 1204.5105.

[36] J. de Boer and J. I. Jottar, "Thermodynamics of Higher Spin Black Holes in $\mathrm{AdS}_{3}$," 1302.0816 .

[37] S. de Buyl, S. Detournay, G. Giribet, and G. S. Ng, "Baby de Sitter Black Holes and $\mathrm{dS}_{3} / \mathrm{CFT}_{2}, " 1308.5569$.

[38] A. Castro and A. Maloney, "The Wave Function of Quantum de Sitter," JHEP 1211 (2012) 096, 1209.5757.

[39] C. Krishnan, "Quantum Field Theory, Black Holes and Holography," 1011.5875.

[40] J. R. David, M. Ferlaino, and S. P. Kumar, "Thermodynamics of higher spin black holes in 3D," JHEP 1211 (2012) 135, 1210.0284.

[41] G. Gibbons and S. Hawking, "Cosmological Event Horizons, Thermodynamics, and Particle Creation," Phys.Rev. D15 (1977) 2738-2751.

[42] J. M. Bardeen, B. Carter, and S. Hawking, "The Four laws of black hole mechanics," Commun.Math.Phys. 31 (1973) 161-170.

[43] A. Perez, D. Tempo, and R. Troncoso, "Higher spin black hole entropy in three dimensions," JHEP 1304 (2013) 143, 1301.0847.

[44] M. Banados, T. Brotz, and M. E. Ortiz, "Boundary dynamics and the statistical mechanics of the (2+1)-dimensional black hole," Nucl.Phys. B545 (1999) 340-370, hep-th/9802076.

[45] P. Kraus and T. Ugajin, "An Entropy Formula for Higher Spin Black Holes via Conical Singularities," JHEP 1305 (2013) 160, 1302.1583. 